\title{
Effects of adenosine receptor antagonists in MPTP mouse model of Parkinson's disease: mitochondrial DNA integrity
}

\author{
Soha S. Essawy ${ }^{1}$, Mona Kamal Tawfik ${ }^{1}$, Horya Erfan Korayem²
}

${ }^{1}$ Department of Pharmacology, Faculty of Medicine, Suez Canal University, Ismailia, Egypt ${ }^{2}$ Department of Histology, Faculty of Medicine, Suez Canal University, Ismailia, Egypt

Submitted: 19 March 2015

Accepted: 19 May 2015

Arch Med Sci 2017; 13, 3: 659-669

DOI: https://doi.org/10.5114/aoms.2017.67284

Copyright $\odot 2017$ Termedia \& Banach

\section{Abstract}

Introduction: In Parkinson's disease (PD), compelling data indicate a functional link between adenosine/dopamine receptors and the progression of the neurodegenerative process. The present study was carried out to evaluate the effect of the non-selective adenosine receptor (ADR) antagonist caffeine, as well as the selective antagonists 8-cyclopentyl-1,3-dipropylxanthine (DPCPX), an ADRsA antagonist, and ((E)-1,3-diethyl-8-(3,4-dimethoxystyryl)-7-methyl-3,7-dihydro-1H-purine-2,6-dione) (KW-6002), an ADRsA antagonist, on the prevention of 1-methyl-4-phenyl-1,2,3,6-tetrahydropyridine (MPTP)-induced Parkinsonism in mice.

Material and methods: Mice were allocated to five groups: group I - control group; group II: MPTP group, received four injections of MPTP $(20 \mathrm{mg} / \mathrm{kg}$, i.p. $)$ at $2 \mathrm{~h}$ intervals; groups III, IV, V: received MPTP and i.p. caffeine $(20 \mathrm{mg} / \mathrm{kg} /$ day) or DPCPX (5 mg/kg/day) or KW-6002 (10 mg/kg/day) starting one week before MPTP injection and continuing for 2 weeks.

Results: Therapy with caffeine or KW-6002 not only led to the reversibility of movement dysfunction and increased the concentrations of dopamine and ATP levels $(p<0.05)$, but also, ameliorates the dopaminergic neuron loss and restored the mtDNA and nDNA integrity $(p<0.05)$. Furthermore, in passive avoidance test, caffeine and DPCPX significantly $(p<0.05)$ reversed the MPTP-induced memory deficits, whereas the specific ADRsA2A antagonist did not.

Conclusions: The current results provide evidence that blockade of both $\mathrm{ADRsA}_{1}$ and $\mathrm{ADRSA}_{2 \mathrm{~A}}$ has therapeutic implications in alleviating MPTP-induced motor and cognitive dysfunction and might be a promising candidate for treatment of PD.

Key words: parkinsonism, MPTP, caffeine, DPCPX, KW-6002.

\section{Introduction}

Parkinson's disease (PD) is a neurodegenerative disorder characterized by progressive degeneration of dopaminergic neurons in the substantia nigra pars compacta (SNpc) [1]. Evidence suggests that age-related neurodegeneration, genetic constitution, toxin exposure, oxidative stress, inflammation and apoptosis play major roles in promoting neurodegeneration [2, 3]. In addition, mitochondrial dysfunction has been implicated, as decreased activity of complex I of the electron transport chain (ETC) and mitochondrial DNA (mtDNA) deletions were reported in PD patients [4].

\author{
Corresponding author: \\ Mona Kamal Tawfik PhD \\ Department \\ of Pharmacology \\ Faculty of Medicine \\ Suez Canal University \\ 002-064 Ismailia, Egypt \\ Phone: 002-0122-2719473 \\ E-mail: dmon_kamal@yahoo. \\ com
}


The MPTP mouse model replicates features of PD. 1-methyl-4-phenylpyridinium ion (MPP+), an MPTP metabolite, inhibits complex I of mitochondrial ETC, leading to selective loss of dopaminergic neurons in the SNpc and motor impairment in rodents [5].

Adenosine in the central nervous system coordinates responses to dopamine and other neurotransmitters that are responsible for motor function, mood, learning and memory [6]. Among the four $G$ protein-coupled ADRs, $A_{1}, A_{2 A}, A_{2 B}$, and $A_{3}, A_{1}$ and $A_{2 A}$ are predominantly expressed in brain. In the striatum, ADRsA appear to be localized pre-synaptically on dopamine axon terminals where they inhibit dopamine release [7]. Additionally, striatal $\mathrm{ADRsA}_{2 \mathrm{~A}}$ are highly expressed in medium spiny neurons of the indirect efferent pathway, where they heteromerize with dopamine $D_{2}$ receptors [8].

Caffeine is a non-selective adenosine receptor antagonist, with reported high affinities for $\mathrm{ADRs}_{1}$, $A_{2 A}$, and $A_{2 B}$ [9]. Several animal models of PD have shown that caffeine strongly interacts with the central dopaminergic systems, mimicking and potentiating the behavioral effects of dopamine receptor agonists [10]. The biological basis of the putative neuroprotective effect of caffeine is not completely understood [11]. It was suggested that both striatal $A D R s A_{1}$ and $A_{2 A}$ are involved in the motor-activating effects of caffeine [12]. In contrast, other experiments indicated that $A_{1}$ receptor contributes to the stimulatory but not the inhibitory motor-activating effects of caffeine [13]. Moreover, while drug discrimination experiments support a key role of $A D R s A_{1}$, but not $A_{2 A}$, in the stimulant effects of caffeine in $P D$, there was found a negative correlation between $A_{1}$ blocking and $\mathrm{PD}[9,14]$. On the other hand, it was proved that blockade of $A D R s A_{2 A}$ facilitates $D_{2}$ receptor function and enhances motor symptoms in PD [15].

Considering that antagonism of both ADRsA $_{1}$ and $A_{2 A}$ would be synergistic and that the relationship between adenosine blocking and PD is still controversial, the present study was conducted to elucidate the possible role of the non-selective competitive antagonist caffeine, as well as specific pharmacologic antagonists - DPCPX, an ADRsA $_{1}$ antagonist, and KW-6002, a specific ADRsA ${ }_{2 A}$ antagonist - in the MPTP mouse model of PD.

\section{Material and methods}

\section{Animals}

Fifty male Swiss albino mice, weighing 20-25 g, were used. Mice were purchased from The National Centre of Research (Cairo, Egypt) and housed in clean, well-ventilated, stainless steel cages under controlled conditions $\left(25 \pm 2^{\circ} \mathrm{C}\right.$, constant relative humidity and reversed dark/light cycle). The cage substrate was replaced each day with food and tap water ad libitum during the study. All experimental procedures were approved by the institutional animal care and use committee at the Suez Canal University following internationally accepted guidelines (NIH): Guide for the Care and Use of Laboratory Animals.

\section{Chemicals and drugs}

MPTP hydrochloride, caffeine, DPCPX and KW6002 were purchased from Sigma-Aldrich (MO, USA). MPTP and caffeine were dissolved in normal saline, while DPCPX and KW-6002 were dissolved in dimethyl sulfoxide (DMSO), and then suspended in $0.3 \%$ Tween-80. DMSO and Tween-80 were purchased from the Egyptian International Pharmaceutical Industrial Company (EIPICO).

\section{Induction of experimental parkinsonism}

Parkinsonism was induced by 4 intraperitoneal (i.p.) doses of MPTP $(20 \mathrm{mg} / \mathrm{kg})$ at 2-hour intervals [16]. Pharmacological treatment started one week before MPTP injection for 2 weeks. In the MPTP model, dopamine cell loss stabilizes 5-7 days after injection [17].

\section{Experimental protocol}

Mice were randomly allocated to 5 equal groups:

- group I (control group): received 4 i.p. injections of normal saline ( $2 \mathrm{ml} / \mathrm{kg}$ ) parallel to MPTP;

- group II (MPTP group): received normal saline

(2 $\mathrm{ml} / \mathrm{kg} /$ day, i.p.) starting one week before MPTP, for 2 weeks;

- group III (caffeine group): received caffeine (20 mg/kg/day/i.p.) [14];

- group IV (DPCPX group): received DPCPX (5 mg/ kg/day/i.p.) [14];

- group V (KW-6002 group): received KW-6002 (10 mg/kg/day/i.p.) [8].

\section{Functional assessment}

At the end of the experiment, motor and cognitive functions were assessed.

\section{Stride length quantitative gait analysis test}

Mice were habituated to the apparatus: an illuminated open field $(60 \times 60 \times 40 \mathrm{~cm})$, with a runway (4.5 cm wide, $42 \mathrm{~cm}$ long, $12 \mathrm{~cm}$ high) leading out into a dark box $(20 \times 17 \times 10 \mathrm{~cm})$. Stride lengths were measured by wetting animals' foreand then hind paws with ink and letting them trot on a strip of paper ( $4.5 \mathrm{~cm}$ wide, $40 \mathrm{~cm}$ long) down the brightly lit runway towards the dark box. The mean of the three longest stride lengths were measured manually [18]. 


\section{Grid walking test}

Mice were placed on a wire grid $(330 \mathrm{~mm}$ diameter, $15 \mathrm{~mm} \times 15 \mathrm{~mm}$ grid squares) and allowed to freely explore for $3 \mathrm{~min}$. The mice were videotaped and later the percentage of foot slips out of the first 50 steps taken was scored [19].

\section{Open field test}

The open-field arena $(113 \times 113 \times 44 \mathrm{~cm})$ was made of dark glass. The floor was painted with white lines that formed a $5 \times 5 \mathrm{~cm}$ pattern. Mice were introduced in the open field arena and observed for 5 min sessions. The horizontal movement (ambulation: the number of squares crossed, number of stops and mobility duration) and the vertical movement (rearing) were registered [20].

\section{Assessment of cognitive function (passive avoidance task)}

Memory impairment was tested by a passive avoidance task. On day 0 (learning trial) the mice were placed in an illuminated box $(40 \times 40 \times$ $30 \mathrm{~cm})$ connected to a dark box $(40 \times 40 \times 30 \mathrm{~cm})$. Entrance to the dark box was punished by an electric foot shock (2 s $0.7 \mathrm{~mA}$ ). Twenty-four hours later, mice were put into the illuminated box and observed for $180 \mathrm{~s}$. Time elapsing before entrance to the dark box was recorded [21].

\section{Processing of the brain}

After functional assessment, mice were anesthetized by i.p. thiopental sodium (50 mg/kg) and killed by decapitation [22]. Brains were dissected and washed with ice-cold saline. The SNpc was dissected [23]. The SN from the right hemisphere was frozen at $-80^{\circ} \mathrm{C}$ and used to detect dopamine, adenosine triphosphate (ATP) and extraction of mtDNA and nuclear DNA (nDNA). The SN from the left hemisphere was prepared for histopathological examination.

\section{Biochemical analysis}

\section{Determination of dopamine}

Frozen tissue was homogenized, and then centrifuged at $10000 \times g$ for $1 \mathrm{~min}$. The supernatant was removed and filtered. Levels of dopamine were measured using reverse-phase high performance liquid chromatography (HPLC) coupled to electrochemical detection (ECD) [24].

\section{Determination of ATP}

The ATP was extracted from the tissues with $10 \mathrm{ml}$ of $0.6 \mathrm{M}$ perchloric acid in an ice bath for $1 \mathrm{~min}$ [25]. The extraction mixture was centrifuged for $10 \mathrm{~min}$ at $6000 \times \mathrm{g}$ and $4^{\circ} \mathrm{C}$, and $6 \mathrm{ml}$ of the supernatant was taken and quickly neutralized to $\mathrm{pH}=6.5$ with $1 \mathrm{M} \mathrm{KOH}$ solution. The neutralized supernatant was then allowed to stand for $30 \mathrm{~min}$ in an ice bath to precipitate most of the potassium perchlorate, which was removed by paper filtration. The filtrate solution was filtered again through a $0.45 \mu \mathrm{m}$ filter. The final filtrate solution was made up to $8 \mathrm{ml}$ and then stored at $-30^{\circ} \mathrm{C}$ prior to the analysis. The concentration of ATP was determined as described previously [24].

\section{Assessment of mtDNA integrity}

\section{Isolation of mitochondria}

Mitochondria were extracted by differential centrifugations [26]. One part (100 mg) of tissue was homogenized in $0.25 \mathrm{M}$ sucrose in 0.7 M Tris$\mathrm{HCl}$ buffer $(\mathrm{pH}=7.4)$ at $1 \mathrm{~g}$ of tissue and $9 \mathrm{ml}$ of Tris-sucrose. EDTA was added to aid disruption of cells. Tissue homogenate was spun at $2500 \times g$ for $10 \mathrm{~min}$ to remove nuclei and unbroken cells. Supernatant fluid was decanted into centrifuge tubes and spun at $10000 \times g$ for $10 \mathrm{~min}$ to form a primary mitochondrial pellet. Supernatant fluid was decanted and the pellet was gently resuspended in $10 \mathrm{ml}$ Tris-sucrose for washing. The pellet was recentrifuged and the supernatant fluid was decanted. This washing cycle was repeated several times to improve the degree of mitochondrial purity. The final mitochondrial pellet was resuspended (1 $\mathrm{ml}$ of Tris-sucrose/1 $\mathrm{g}$ of original sample).

\section{Isolation of mtDNA}

The mtDNA samples were subjected to $1 \%$ agarose gel electrophoresis at $4 \mathrm{~V} / \mathrm{cm}$ using TAE solution (40 mM Tris-acetate, $\mathrm{pH}$ 8.0, 1 mM EDTA) as a running buffer. The gel was stained with $0.5 \mu \mathrm{g} / \mathrm{ml}$ ethidium bromide and photographed using the G:Box Gel Documentation system (Syngene, USA) [27]. Furthermore, mtDNA quantity and purity were determined using a NanoDrop 1000 spectrophotometer V3.7 (Thermo Fisher Scientific Inc., Wilmington, DE, USA).

\section{DNA laddering assay}

Endogenous endonucleases - a group of enzymes that cleave double-stranded DNA in the linker region between nucleosomes, and generate mono- and oligonucleosomes of 180 bp or multiples - are one of the characteristics of apoptosis. To assess endonuclease-dependent ladder-like DNA fragmentation by gel electrophoresis, genomic DNA was extracted from the tissue by $100 \mathrm{bp}$ DNA Ladder, Cat. No. "M-214S" (Germany) according to the manufacturer's guidelines then loaded onto agarose gel (15 $\mu \mathrm{g} /$ lane). DNA laddering 
was determined by constant voltage mode electrophoresis (in a large submarine at $4 \mathrm{~V} / \mathrm{cm}$, for $4 \mathrm{~h}$ ) on a $1.5 \%$ agarose gel containing $0.5 \mu \mathrm{g} /$ $\mathrm{ml}$ ethidium bromide. A $1 \mathrm{kbp}$ ladder served as a DNA base pair marker [28]. Gels were visualized by the G:Box Gel Documentation system (Syngene, USA).

\section{Histopathology and immunohistochemistry}

The SN of the left hemispheres from each brain was stained with hematoxylin \& eosin ( $\mathrm{H}$ \& E), cresyl violet for Nissl granules and phosphotung stic acid hematoxylin (PTAH) for dendritic arborizations. Immunostaining was performed using rabbit monoclonal primary antibodies (R\&D Systems) against neuronal tyrosine hydroxylase (TH). Using the light microscope, followed by an image analyzer, histological sections of SNpc were examined for quantitative assessment of the neuronal population and percentage in $\mathrm{TH}$ immunostained sections.

\section{Statistical analysis}

Data were expressed as mean \pm SEM and analyzed using the Statistical Package for the Social Sciences (SPSS). The difference of mean values among groups was assessed using one-way analysis of variance (ANOVA) followed by Bonferroni's multiple comparisons test. All $p$-values $<0.05$ were considered significant.

\section{Results}

\section{Functional assessment}

\section{Stride length quantitative gait analysis test}

The present study showed that MPTP was associated with a significant $(p<0.05)$ difference between mice forelimbs' and hind limbs' stride

A

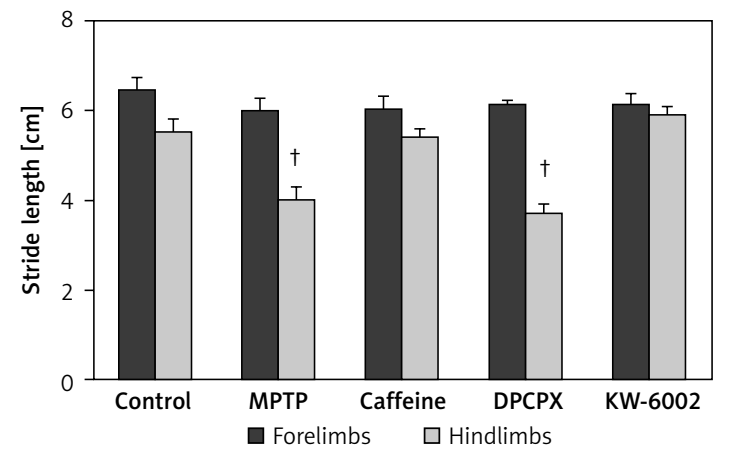

length in comparison with the control group (Figure $1 \mathrm{~A}$ ). Only caffeine and KW-6002 abolished the difference in the stride length compared to the MPTP group (Figure $1 \mathrm{~A}, p<0.05$ ).

\section{Grid walking test}

The MPTP developed significant foot slip errors in the grid walking test. Treatment with either caffeine or KW-6002 ameliorated the percentage of foot slip errors compared to MPTP (Figure $1 \mathrm{~B}$, $p<0.05)$. Importantly, an insignificant improvement in foot slip errors was recorded in mice that received DPCPX (Figure $1 \mathrm{~B}, p>0.05$ ).

\section{Open field test}

Mice treated with MPTP showed poor mobility in the open field test revealed by reduction in the ambulation, number of stops, mobility duration as well as rearing frequency (Figures $2 \mathrm{~A}-\mathrm{D}, p<0.05$ ) in comparison with the control group. Treatment with either caffeine or KW-6002 significantly ameliorated the poor mobility compared to MPTP (Figures $2 \mathrm{~A}-\mathrm{D}, \mathrm{p}<0.05)$.

\section{Cognitive function}

Figure 3 shows that latency time in the passive avoidance task was lower $(p<0.05)$ in the MPTP group compared to the control group. Only caffeine and DPCPX can improve latency time compared to MPTP $(p<0.05)$.

\section{Biochemical analysis \\ Dopamine and ATP levels}

MPTP-treated mice showed a significant reduction in dopamine and ATP levels compared to the control group. Treatment with caffeine or KW6002 increased these parameters in comparison

B

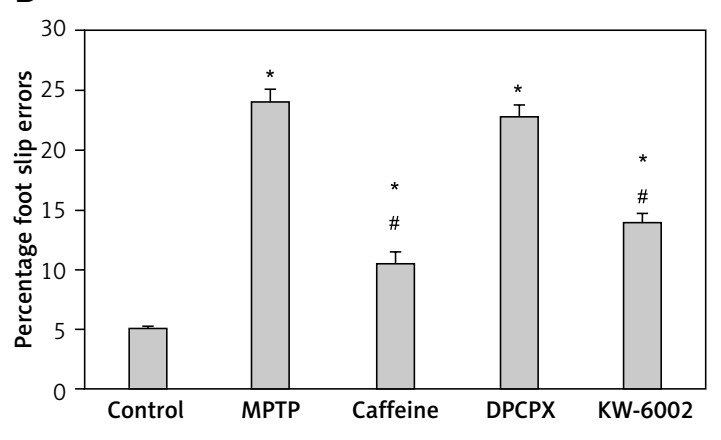

Figure 1. Stride length (A) and percentage of foot slips in grid walking (B). Caffeine and KW-6002 ameliorated the difference between forelimbs' and hind limbs' stride length and decreased the percentage of foot slip errors compared to the MPTP group (A \& B)

MPTP - 1-methyl-4-phenyl-1,2,3,6-tetrahydropyridine. DPCPX - 8-cyclopentyl-1,3-dipropylxanthine, KW-6002 - [(E)-1,3-diethyl8-(3,4-dimethoxystyryl)-7-methyl-3,7-dihydro-1H-purine-2,6-dione]. Results are mean \pm SEM and were analyzed using one-way ANOVA and Bonferroni post-hoc test; ${ }^{\dagger} p<0.05$ compared to forelimbs' stride length in MPTP group; ${ }^{*} p<0.05$ compared to control group; $" p<0.05$ compared to MPTP group. 
A

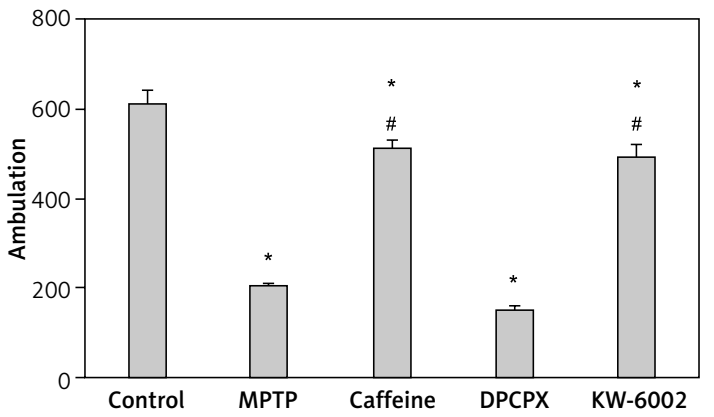

$\mathrm{C}$

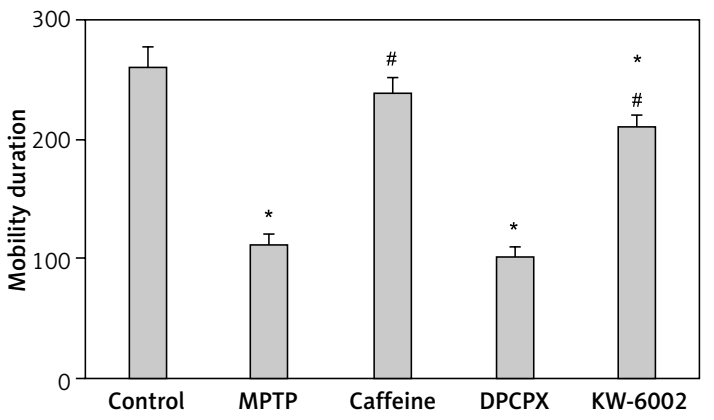

B

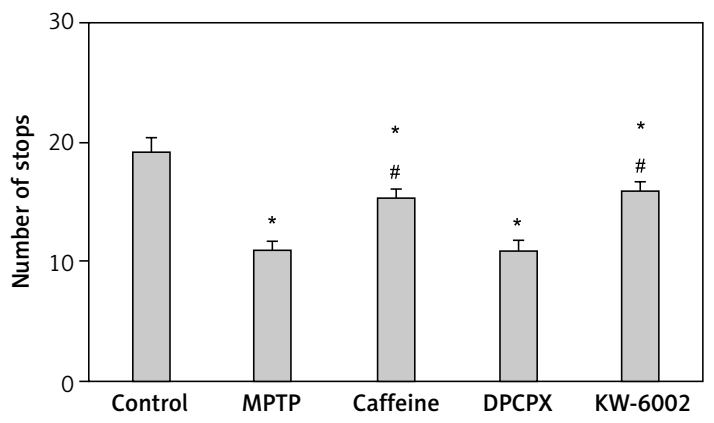

D

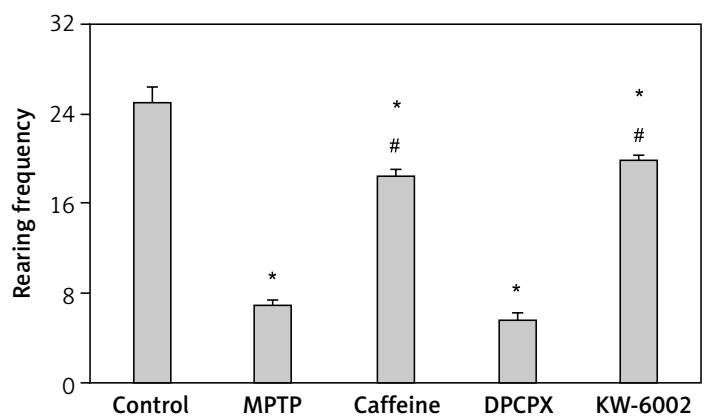

Figure 2. Motor performance in open field test. Mice were observed for the ambulation (A), number of stops (B), mobility duration (C) and rearing frequency (D). Caffeine or KW-6002 improved MPTP-induced motor dysfunction MPTP - 1-methyl-4-phenyl-1,2,3,6-tetrahydropyridine, DPCPX - 8-cyclopentyl-1,3-dipropylxanthine, KW-6002-[(E)-1,3-diethyl8-(3,4-dimethoxystyryl)-7-methyl-3,7-dihydro-1H-purine-2,6-dione]. Results are mean \pm SEM and were analyzed using one-way ANOVA and Bonferroni post-hoc test; ${ }^{*} p<0.05$ compared to control group; ${ }^{*} p<0.05$ compared to MPTP group.

with MPTP (Figures 4 A, B, $p<0.05$ ). Notably, an insignificant improvement in dopamine or ATP levels was detected in mice that received DPCPX compared to MPTP (Figures $4 \mathrm{~A}, \mathrm{~B}, p>0.05$ ).

\section{Mitochondrial DNA integrity and fragmentation of nuclear DNA}

In the control group, the intact form of mtDNA was electrophoresed as a major band (lanes 1 , 2). Administration of MPTP markedly decreased the amounts of intact mtDNA (lane 5). Treatment with DPCPX resulted in an insignificant increase in the amounts of intact mtDNA (lane 6); however, mtDNA from mice treated with caffeine (lanes 7, 8) and KW-6002 (lanes 3, 4) were electrophoresed in their intact form (Figure $5 \mathrm{~A}$ ). MPTP resulted in a marked decrease in intact nDNA amount (Figure $5 \mathrm{~B}$, lanes 3,4$)$. Moreover, a dramatic degradation of DNA was observed in the DPCPX group (lanes 9, 10), characterized by mixed smearing and laddering. No appreciable fragmentation of nDNA was found in animals treated with caffeine (lanes 5, 6) or KW-6002 (lanes 7, 8). In the MPTP-treated group, a decline was observed in mtDNA tissue content, as compared to that of the control group (Figure $5 \mathrm{C}, p<0.05$ ). However, improvement of the mtDNA content was observed upon treatment with caffeine or KW-6002 (Figure 5 C, $p<0.05$ )

\section{Histopathological examination and immunostaining}

H\&E stained SNpc of the control group showed a cell-rich region with abundant large multipolar neurons with vesicular nuclei and the surrounding neutrophil. Pars compacta cells - in sections from MPTP and DPCPX groups - appeared widely lost or angulated and shrunken with dark stained cyto-

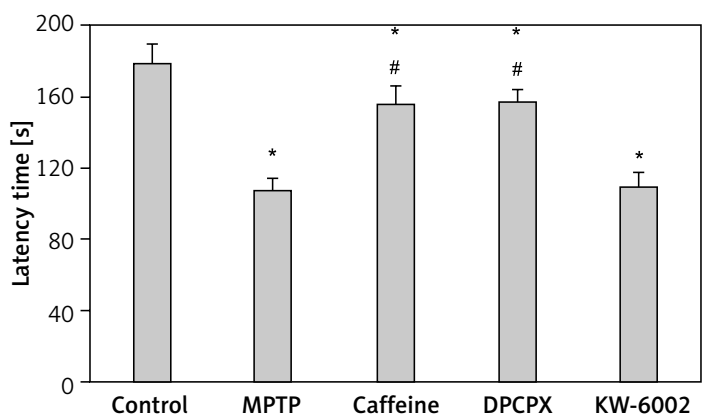

Figure 3. Cognitive function in passive avoidance task. Mice were observed for the latency time for 180 s. Treatment with caffeine and DPCPX improved latency time compared to MPTP group

MPTP - 1-methyl-4-phenyl-1,2,3,6tetrahydropyridine, DPCPX 8-cyclopentyl-1,3-dipropylxanthine, KW-6002 - [(E)-1,3diethyl-8-(3,4-dimethoxystyryl)-7-methyl-3,7-dihydro$1 \mathrm{H}$-purine-2,6-dione]. Results are mean \pm SEM and were analyzed using one-way ANOVA and Bonferroni posthoc test; ${ }^{*} p<0.05$ compared to control group; ${ }^{*} p<0.05$ compared to MPTP group. 
A

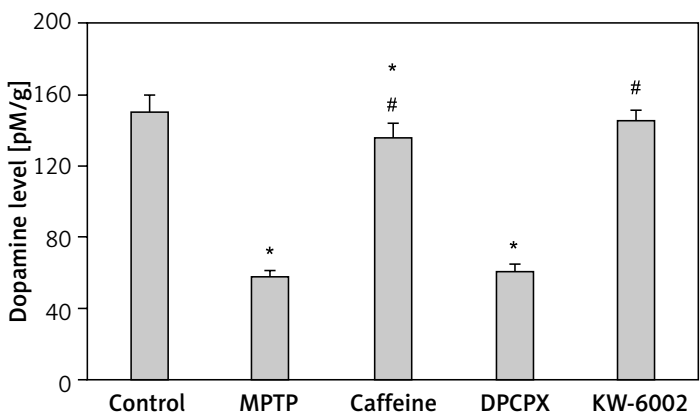

B

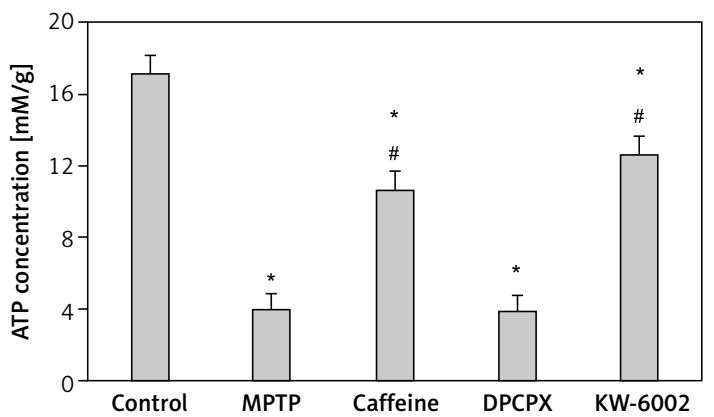

Figure 4. Dopamine (A) and ATP (B) levels in experimental groups. Treatment with caffeine and KW-6002 increased dopamine and ATP levels compared to MPTP

MPTP - 1-methyl-4-phenyl-1,2,3,6-tetrahydropyridine, DPCPX - 8-cyclopentyl-1,3-dipropylxanthine, KW-6002-[(E)-1,3-diethyl8-(3,4-dimethoxystyryl)-7-methyl-3,7-dihydro-1H-purine-2,6-dione]. Results are mean \pm SEM and were analyzed using one-way ANOVA and Bonferroni post-hoc test; ${ }^{*} p<0.05$ compared to control group; ${ }^{*} p<0.05$ compared to MPTP group.

A

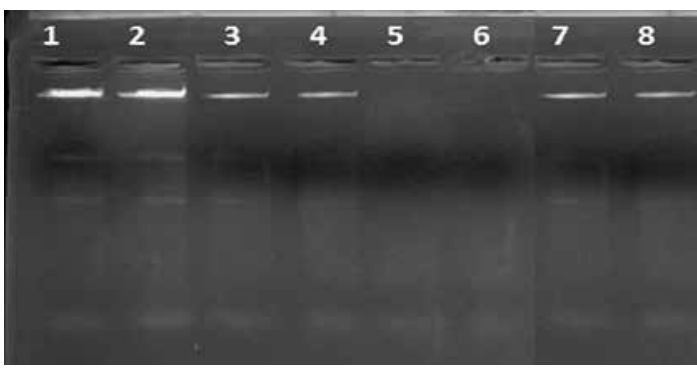

C

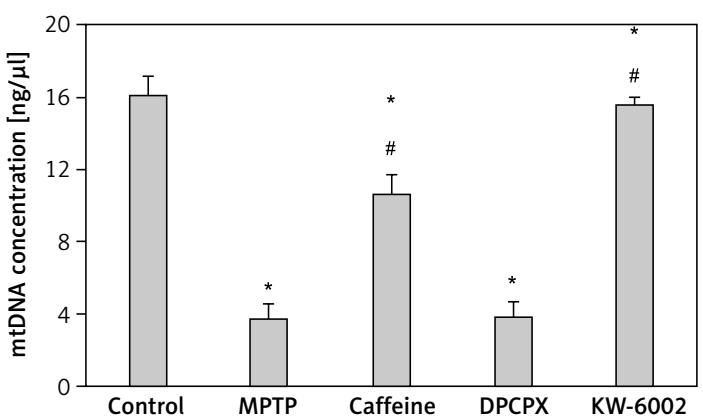

\section{B}

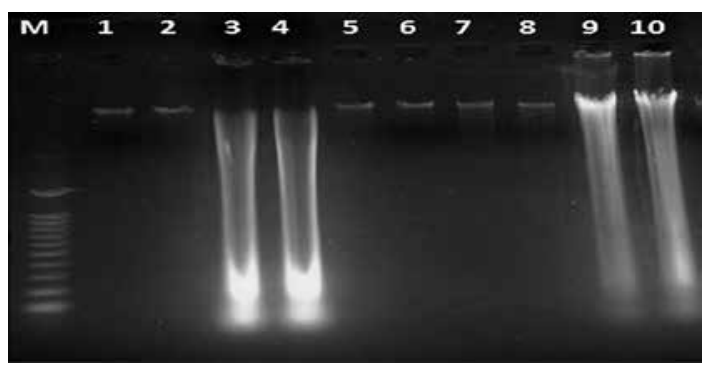

Figure 5. Mitochondrial DNA (mtDNA) and nuclear DNA (nNDA) in experimental groups. A - mtDNA integrity. Lanes 1, 2 - intact mtDNA in control group; lanes 3, 4-mtDNA in KW-6002 group; lanes 5, 6- mtDNA in MPTP and DPCPX respectively; lanes 7, 8 - mtDNA in caffeine group. B - nDNA integrity, lane $M-1$ kb DNA ladder; lanes 1, 2 - intact nDNA in control group; lanes 3, 4 - nDNA in MPTP group; lanes 5, 6-nDNA in caffeine group; lanes 7, 8 - nDNA in KW-6002 group, lanes 9, 10 - nDNA in DPCPX group. C - mtDNA concentrations. MPTP-induced mtDNA damage was significantly decreased upon administration of caffeine or KW-6002

MPTP - 1-methyl-4-phenyl-1,2,3,6-tetrahydropyridine, DPCPX - 8-cyclopentyl-1,3-dipropylxanthine, KW-6002-[(E)-1,3-diethyl8-(3,4-dimethoxystyryl)-7-methyl-3,7-dihydro-1H-purine-2,6-dione]. Results are mean \pm SEM and were analyzed using one-way ANOVA and Bonferroni post-hoc test. ${ }^{*} p<0.05$ compared to control group; ${ }^{*} p<0.05$ compared to MPTP group.

plasm and a deep stained nucleus. In caffeine and KW-6002-treated animals, SNpc showed scattered large multipolar cells and surrounding neutrophil similar to that of the control group. Caffeine and KW-6002 resulted in a significant $(p<0.05)$ reduction in the percent necrotic change in Purkinje fibers (Figure $6(I)$ ).

Examination with cresyl fast violet stain showed aggregates of purple Nissl granules within the perikarya of SNpc in the control group. The mean optical density of Nissl granules was 0.33 \pm 0.02 . In the MPTP group, there was a significant decrease of the Nissl granules in the perikarya of SNpc cells $(0.13 \pm 0.01)$ compared to the control group that was not improved with DPCPX (0.18 \pm 0.01 ). However, caffeine and KW-6002 revealed increased purple Nissl granules of nigral neurons with mean optical density $0.29 \pm 0.02$ and 0.27 \pm 0.02 respectively compared to MPTP (Figure 6 (II)), $p<0.05)$. 

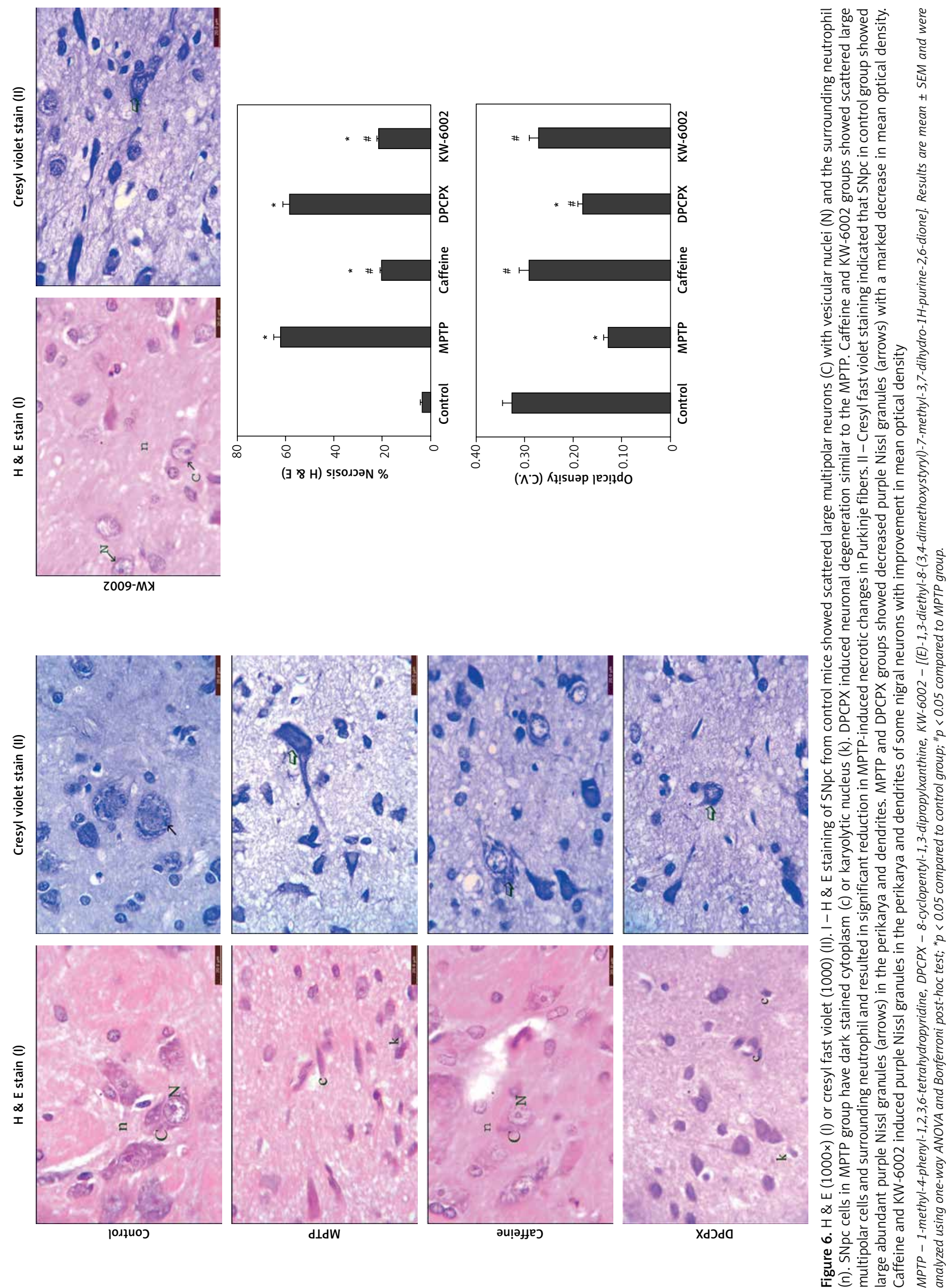

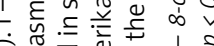

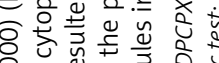

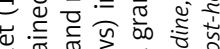

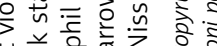

要需

ฟे

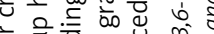

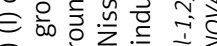

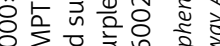

들

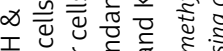

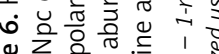

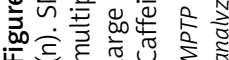


Using PTAH stain, delicate blue dendritic arborizations of SNpc cells (Figure 7 (I)) in the control group appeared with mean dendrite length 0.52 $\pm 0.04 \mu \mathrm{m}$.

PTAH stain in the MPTP group showed a marked decrease of SNpc cells' dendritic arborizations with a significant decrease in mean dendrite length $0.3 \pm 0.03 \mu \mathrm{m}$ (Figure $7(\mathrm{I}), p<0.05$ ). The dendritic arborizations of cells were significantly increased in length upon treatment with caffeine or KW-6002 compared to the MPTP group with mean dendrite length $0.48 \pm 0.03 \mu \mathrm{m}$ and 0.47 $\pm 0.02 \mu \mathrm{m}$, respectively.

All control mice revealed uniform positive brownish staining of the soma and processes of all SNpc cells (Figure 7 (II)) with mean optical density of TH activity $0.87 \pm 0.02$. In MPTP group, positive staining for TH was decreased $(p<0.05)$ in $100 \%$ of animals with mean optical density of $\mathrm{TH}$ activity $0.62 \pm 0.04$. Increased staining of the soma and processes of all SNpc cells was detected in $100 \%$ of animals treated with caffeine or KW-6002 compared to MPTP $(p<0.05)$ with mean optical density of TH activity $0.76 \pm 0.03$ and 0.77 \pm 0.03 respectively (Figure 7 (II)).

\section{Discussion}

Although the pathological hallmark in PD patients is progressive dopaminergic neurodegeneration in the basal ganglia, so far there is no efficient strategy to counteract the progressive death of the dopaminergic neurons of the substantia nigra.

Combining the fact that adenosine receptors participate in the antagonistic interaction between adenosine and dopamine and they have been implicated in motor and cognitive dysfunction $[29,30]$ with the fact that patients experiencing levodopa-induced dyskinesia show increased ADRsA $_{2 A}$ availability in the striatum [31], adenosine receptor antagonists were a logical candidate for study as promising non-dopaminergic therapy of PD. Therefore, the present study was conducted to examine the possible protective effect of the non-selective competitive antagonist caffeine, as well as the specific pharmacologic antagonists DPCPX (ADRsA $)$ and KW-6002 (ADRsA $\left.{ }_{2 A}\right)$, on experimental parkinsonian mice.

The MPTP, a highly selective complex I inhibitor, is known to reproduce the neurochemical, neuropathological, and behavioral features of PD in rodents [32]. In the present study, i.p. MPTP impaired motor function, coordination and cognitive function, together with decreased SNpc dopamine and ATP levels as well as neurodegeneration. Consistent with previous studies emphasizing that in neurotoxic models of parkinsonism, symptoms are detectable when markers of dopaminergic neurons in the SNpc fall below $20-40 \%$ of normal values [15, 33], the current results revealed that neuropathological changes in the SNpc regions were accompanied by reduction in the density of TH-positive fibers as well as a $62 \%$ decrease in SNpc dopamine level. It was emphasized that dopamine depletion in the striatum produces profound deficits in reaction time in rats commonly seen in parkinsonism [34]. In addition, in PD, the basal ganglia input to the thalamus becomes pathological and relay of motor-related cortical inputs is compromised, thereby impairing movements [35]. This could be attributed to the accumulation of $\mathrm{MPP}^{+}$in the mitochondrial matrix, where it binds to complex I and interrupts movement of electrons along the ETC, thus producing reactive oxygen species, and mitochondrial injury associated with a drop in ATP production in susceptible areas of the brain such as the striatum $[36,37]$. In line with this notion, our results demonstrated that MPTP was associated with mitochondrial injury as revealed by reduction of the integrity and amount of intact mtDNA and nDNA, which are targets for the MPTP insult that may contribute to the neurodegenerative process in PD [38].

The interaction of the adenosine/dopamine receptors down-modulates the latter, and their effects on motor activity were described previously [39]. In line with previous studies, the current study confirmed that therapy with caffeine or KW6002 not only reversed the MPTP-induced motor dysfunction and increased the SNpc dopamine and ATP levels, but also ameliorated the dopaminergic neuron loss and restored the mtDNA and nDNA integrity $[8,40]$. Caffeine may increase striatal dopamine via blockade of inhibitory presynaptic ADRsA $_{1}$ [41]. In contrast, Chen et al. [14] suggested that the neuroprotective actions of this non-specific ADRs antagonist were mimicked by multiple $A_{2 A}$-specific antagonists but not by $A_{1}$-specific antagonists. MPTP toxicity was blunted in mice lacking ADRsA $_{2 A}$, confirming their role in facilitating MPTP toxicity and supporting their contribution to the neuroprotective effect of caffeine.

Additionally, ADRsA $A_{2 A}$ are localized in striatal glutamatergic terminals where they are involved in the modulation of glutamate release [42]; activation of $A D R s A_{2 A}$ results in an increased concentration of extracellular glutamate, while the $\mathrm{ADRsA}_{2 \mathrm{~A}}$ antagonists inhibit glutamate release from cells [29, 43]. Moreover, it was emphasized that activation of $A D R s A_{2 A}$ inhibits the release of GABA and the GABA-ergic transmission [44]. Another explanation emphasized that caffeine and $\mathrm{ADRs}_{2 \mathrm{~A}}$ antagonists are competitive monoamine oxidase-B (MAO-B) inhibitors, slowing down the dopamine metabolism and increasing the synaptic level of dopamine [45]. 

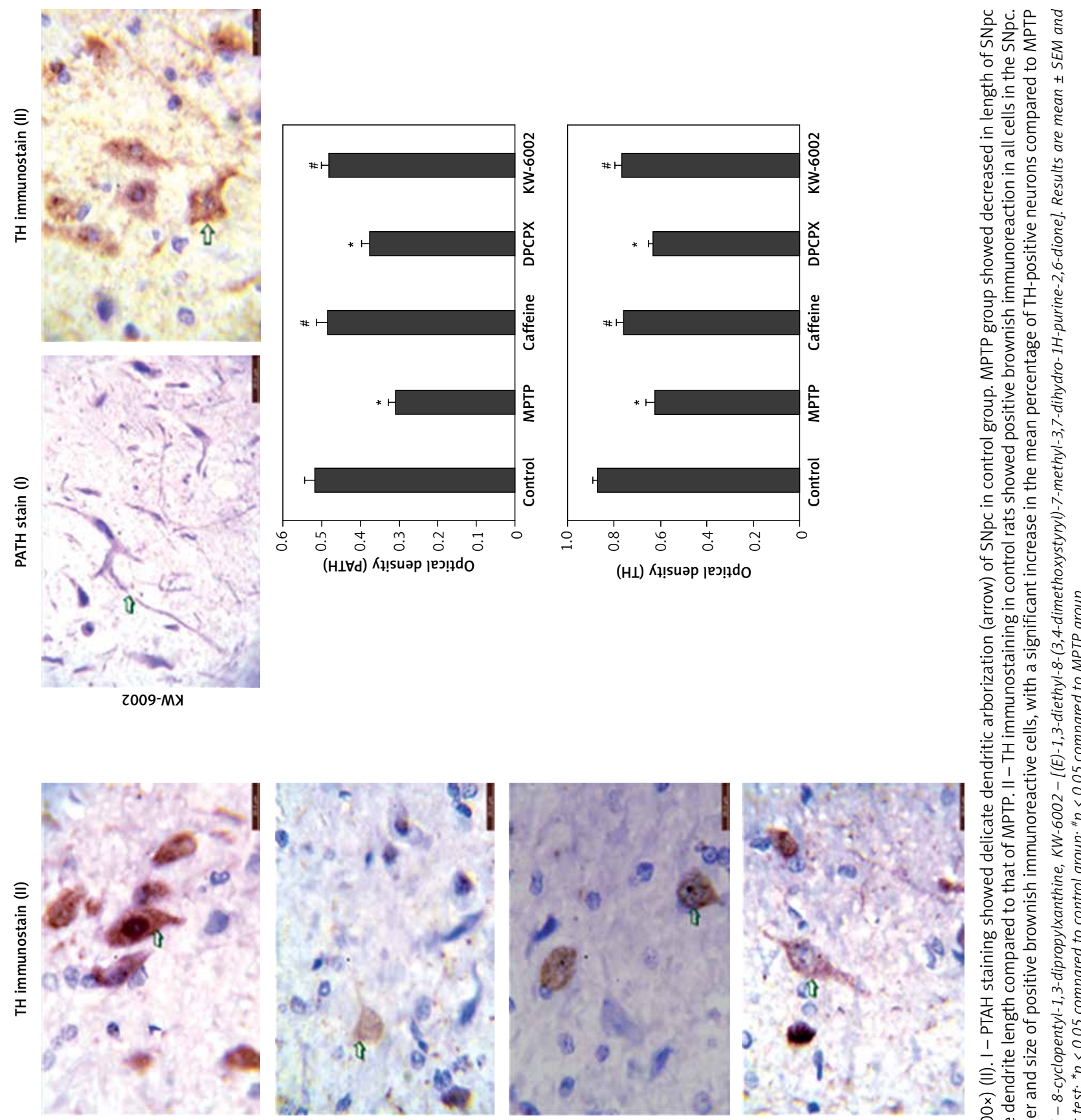

$\stackrel{2}{=} \geq$

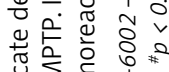

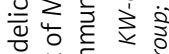

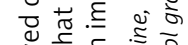

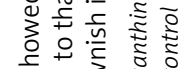

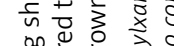

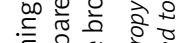

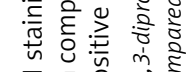

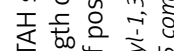

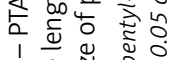

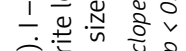

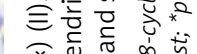

×ᄌ

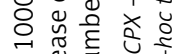
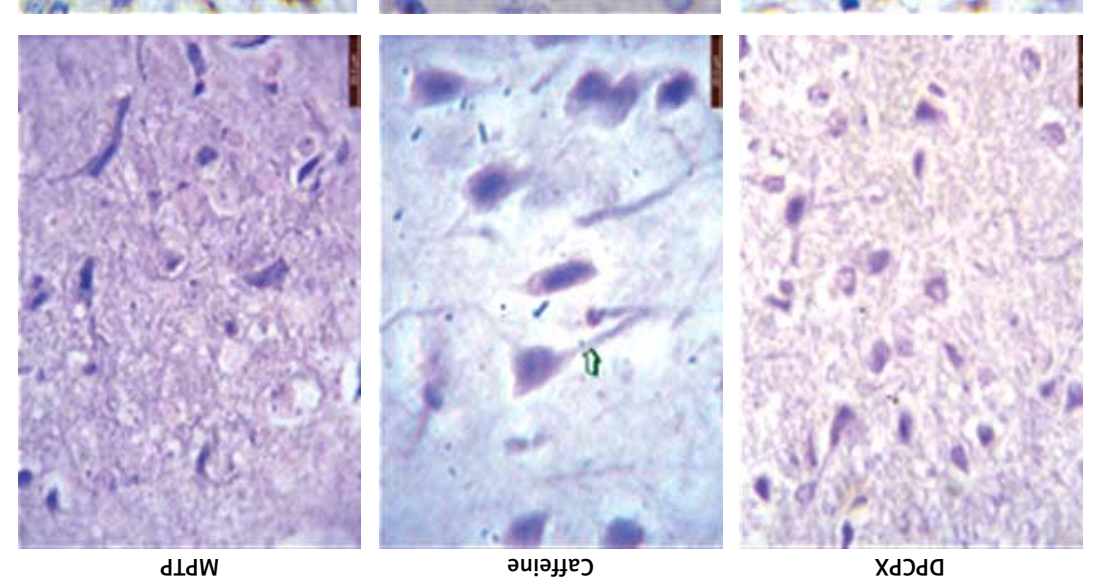

a.

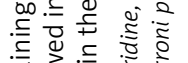

荡

है जิ

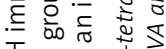

I $\widetilde{0}$ क्षे

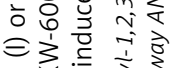

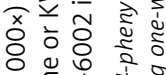

要.

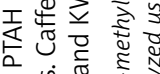

$\sim$ 过

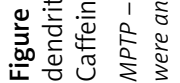


In accordance, DPCPX revealed effectiveness in ameliorating MPTP-induced cognitive dysfunction only, with no effect on motor activity [24]. In contrast to our results, it was also proved that selective ADRsA $A_{1}$ antagonists were able to mimic the effect of caffeine and increased the locomotion in mice [40]. Our observation is supported by the previously reported observation that the neuroprotective action of ADRs antagonists in rat models of PD is coupled to the blockade of ADRsA and not ADRsA ${ }_{1}$ [46].

In parkinsonism, compelling data indicate a functional link between adenosine ADRsA $_{1}$ and cognitive disorders [46]. In the present study, attenuation of the cognitive impairment by both caffeine and the ADRsA, antagonist with no effect of the $\mathrm{ADRSA}_{2 A}$ antagonist confirmed the involvement of $A D R S A_{1}$ in the memory-improving effect. The cognitive effect of caffeine is mostly due to its ability to antagonize ADRsA in the hippocampus and prefrontal cortex, the brain areas involved in cognition [47]. However, the $\mathrm{ADRsA}_{2 \mathrm{~A}}$ are abundant in the striatum and other nuclei of the basal ganglia, where they are always co-localized with the dopaminergic $D_{2}$ receptors [48]. Queiroz et al. [48] emphasized that ADRsA maintain tonic homoeostatic adenosine functions, whereas ADRsA mostly exert their fine-tuning modulation under some pathophysiological situations. Another possible explanation could be that ADRsA activation depresses cholinergic transmission, which is important in learning and memory processes; thus, blockade of ADRsA may indirectly stimulate cholinergic neurotransmission and thereby enhance cognition [48].

In conclusion, both $\mathrm{ADRsA}_{1}$ and $\mathrm{ADRs}_{2 \mathrm{~A}}$ antagonists have a neuroprotective effect, possessing the potential to alleviate MPTP-induced cognitive and motor impairment in an MPTP model of PD possibly by increasing dopamine and ATP levels in addition to restoring mtDNA and nDNA integrity. Hence, the current data provide evidence that blockade of both ADRsA $_{1}$ and ADRsA $_{2 A}$ might have therapeutic implications for neurodegenerative disease such as PD. Further studies are still needed to confirm the possibility of clinical application of such a combination.

\section{Conflict of interest}

The authors declare no conflict of interest.

\section{References}

1. Haleagrahara N, Ponnusamy K. Neuroprotective effect of Centella asiatica extract (CAE) on experimentally induced parkinsonism in aged Sprague-Dawley rats. J Toxicol Sci 2010; 35: 41-7.

2. Lang A, Lozano A. Parkinson's disease (first of two parts). N Engl J Med 1998; 339: 1044-53.
3. Hunot S, Hirsch E. Neuroinflammatory processes in Par kinson's disease. Ann Neurol 2003; 53 Suppl. 3: S49-58.

4. Lin M, Beal M. Mitochondrial dysfunction and oxidative stress in neurodegenerative diseases. Nature 2006; 443: 787-95.

5. Dehay B, Bezard E. New animal models of Parkinson's disease. Mov Disord 2011; 26: 1198-205.

6. Ruby C, Adams C, Knight E, Nam H, Choi D. An essential role for adenosine signaling in alcohol abuse. Curr Drug Abuse Rev 2010; 3: 163-74.

7. Schiffmann S, Fisone G, Moresco R, Cunha R, Ferré S. Adenosine A2A receptors and basal ganglia physiology. Prog Neurobiol 2007; 83: 277-92.

8. Orru M, Bakešová J, Brugarolas $M$, et al. Striatal pre and postsynaptic profile of adenosine $A 2$ receptor antagonist. PLoS One 2011; 6: e16088.

9. Solinas M, Ferré S, Antoniou K, et al. Involvement of adenosine $A_{1}$ receptors in the discriminative-stimulus effects of caffeine in rats. Psychopharmacology (Berl) 2005; 179: 576-86.

10. Cauli O, Morelli M. Caffeine and the dopaminergic system. Behav Pharmacol 2005; 16: 63-77.

11. Popat R, Van Den Eeden S, Tanner C, et al. Coffee, $A_{A D O R}$, and $C_{A 2} P_{1 A 2}$ : the caffeine connection in Parkinson's disease. Eur J Neurol 2011; 18: 756-65.

12. Justinova Z, Ferré S, Barnes C, et al. Effects of chronic caffeine exposure on adenosinergic modulation of the discriminative-stimulus effects of nicotine, methamphetamine, and cocaine in rats. Psychopharmacol J (Berl) 2009; 203: 355-67.

13. Kuzmin A, Johansson B, Gimenez L, Ogren S, Fredholm B. Combination of adenosine $A 1$ and $A 2$ receptor blocking agents induces caffeine-like locomotor stimulation in mice. Eur Neuropsychopharmacol 2006; 16: 129-36.

14. Chen J, Xu K, Petzer J, et al. Neuroprotection by caffeine and $A(2 A)$ adenosine receptor inactivation in a model of Parkinson's disease. J Neurosci 2001; 2: RC143.

15. Barret O, Hannestad J, Alagille D, et al. Adenosine 2A receptor occupancy by tozadenant and preladenant in rhesus monkeys. J Nucl Med 2014; 55: 1712-8.

16. Serra P, Pluchino S, Marchetti B, Desole MS, Miele E. The MPTP mouse model: cues on DA release and neural stem cell restorative role. Parkinsonism Relat Disord 200814 Suppl. 2: s189-93.

17. Jackson-Lewis V, Przedborski S. Protocol for the MPTP mouse model of Parkinson's disease. Nat Protoc 2007; 2: 141-51.

18. Fernagut O, Elsa D, Bertrand L, Francois T. A simple method to measure strides length as an index of nigrostriatal dysfunction in mice. J Neurosci Methods 2002; 113: 123-30.

19. Menet V, Prieto M, Privat A, Ribotta M. Axonal plasticity and functional recovery after spinal cord injury in mice deficient in both glial fibrillary acidic protein and vimentin genes. Proc Natl Acad Sci 2003; 100: 8999-9004.

20. Dodd C, Bloomquist J, Klein B. Consequences of manganese administration for striatal dopamine and motor behavior in 1-methyl-4-phenyl-1,2,3,6-tetrahydropyridine-exposed C57BL/6 mice. Hum Exp Toxicol 2013; 32: 865-80.

21. Borowicz K, Piskorska A, Luszczki J, Czuczwar S. Influence of SIB 1893, a selective mGluR5 receptor antagonist, on the anticonvulsant activity of conventional antiepileptic drugs in two models of experimental epilepsy. Pol J Pharmacol 2003; 55: 735-40. 
22. Vogler G. Anesthesia and analgesia. In: The Laboratory Rat. $2^{\text {nd }}$ ed. Suckow M, Weisbroth S, Franklin C (eds.) Elsevier Academic Press, New York 2006; 627-95.

23. Kozina E, Khaindrava V, Kudrin V, et al. Experimental modeling of functional deficiency of the nigrostriatal dopaminergic system in mice. Ross Fiziol Zh Im I M Sechenova 2010; 96: 270-82.

24. Liu H, Zhang W, Luo X, Ye Y, Zhu X. Paeoniflorin attenuates neuro-inflammation and dopaminergic neurodegeneration in the MPTP model of Parkinson's disease by activation of adenosine $\mathrm{A} 1$ receptor. $\mathrm{Br} J$ Pharmacol 2006; 148: 314-25.

25. Yang N, Ho W, Chen Y, Hu M. A convenient one-step extraction of cellular ATP using boiling water for the luciferin-luciferase assay of ATP. Anal Biochem 2002; 306: 323-7.

26. Chappel J, Hansford R. Subcellular Components. $2^{\text {nd }}$ ed. Butterworths, London 1969.

27. Chang B, Nishikawa M, Sato E, Utsumi K, Inoue M. L-carnitine inhibits cisplatin-induced injury of the kidney and small intestine. Arch Biochem Biophys 2002; 405: 55-64.

28. Sambrook J, Maniatis T, Fritsch, F. Molecular Cloning: A Laboratory Manual. $3^{\text {rd }}$ ed. Cold Spring Harbor Laboratory Press, Cold Spring Harbor: New York 2001.

29. Gołembiowska K, Wardas J, Noworyta-Sokołowska K, Kamińska K, Górska A. Effects of adenosine receptor antagonists on the in vivo LPS-induced inflammation model of Parkinson's disease. Neurotox Res 2013; 24: 29-40.

30. Antonini A, Poewe W. Adenosine $A_{2 A}$ receptor antagonists in Parkinson's disease: still in the running. Lancet Neurol 2014; 13: 748-9.

31. Ramlackhansingh A, Bose S, Ahmed I, Turkheimer F, Pavese N, Brooks D. Adenosine 2A receptor availability in dyskinetic and nondyskinetic patients with Parkinson disease. Neurol J 2011; 76: 1811-6.

32. Sy H, Wu S, Wang W, et al. MPTP-induced dopaminergic degeneration and deficits in object recognition in rats are accompanied by neuroinflammation in the hippocampus. Pharmacol Biochem Behav 2010; 95: 158-65.

33. McGeer P, McGeer E. Inflammation and neurodegeneration in Parkinson's disease. Parkinsonism Relat Disord 2004; 101: S3-7.

34. Abd-El Gawad H, Abdallah D, El-Abhar H. Rotenone-induced Parkinson's like disease: modulating role of coenzyme Q10. J Biol Sci 2004; 4: 568-74.

35. Altuğ F, Acar F, Acar G, Cavlak U. The effect of brain stimulation on subthalamic nucleus surgery on gait and balance performance in Parkinson disease. A pilot study. Arch Med Sci 2014; 10: 733-8.

36. Khan F, Sen T, Maiti A, Jana S, Chatterjee U, Chakrabarti S. Inhibition of rat brain mitochondrial electron transport chain activity by dopamine oxidation products during extended in vitro incubation: implications for Parkinson's disease. BBA Mol Bas Dis 2005; 1741: 65-74.

37. Abo-Grisha N, Essawy S, Abo-Elmatty DM, Abdel-Hady Z. Effects of intravenous human umbilical cord blood CD34+ stem cell therapy versus levodopa in experimentally induced Parkinsonism in mice. Arch Med Sci 2013; 9: 1138-51.

38. Hoang T, Choi D, Nagai M, et al. Neuronal NOS and cyclooxygenase- 2 contribute to DNA damage in a mouse model of Parkinson disease. Free Radic Biol Med 2009; 47: 1049-56.

39. Franco R, Ferré S, Agnati L, et al. Evidence for adenosine/ dopamine receptor interactions. Indications for heterom erization. Neuropsychopharmacol J 2000; 23: S50-9.
40. Górska A, Gołembiowska K. The role of adenosine $A_{1}$ and $A_{2 A}$ receptors in the caffeine effect on MDMA-induced DA and5-HT release in the mice striatum. Neurotox Res 2015; 27: 229-45.

41. Ciruela F, Casadó V, Rodrigues R, et al. Presynaptic control of striatal glutamatergic neurotransmission by adenosine A1-A2A receptors heteromers. J Neurosci 2006; 26: 2080-7.

42. Gołembiowska K, Dziubina A, Kowalska M, Kamińska K. Effect of adenosine A2A receptor antagonists on L-DOPA induced hydroxyl radical formation in rat striatum. Neurotox Res 2009; 15: 155-66.

43. Cieślak $M$, Komoszyński $M$, Wojtczak A. Adenosine $A_{2 A}$ receptors in Parkinson's disease treatment. Purinergic Signal J 2008; 4: 305-12.

44. Castagnoli N, Petzer J, Steyn S, et al. Monoamine oxidase $B$ inhibition and neuroprotection. Studies on selective adenosine $A_{2 A}$ receptor antagonists. Neurol J 2003; 61: S62-8.

45. Ikeda K, Kurokawa M, Aoyama S, Kuwana Y. Neuroprotection by adenosine $\mathrm{A} 2 \mathrm{~A}$ receptor blockade in experimental models of Parkinson's disease. Neurochem J 2002; 80: 262-70.

46. Ribeiro J, Sebastião A. Caffeine and adenosine. J Alzheimers Dis 2010; 20: S3-15.

47. Mihara T, Mihara K, Yarimizu J, et al. Pharmacological characterization of a novel, potent adenosine $A_{1}$ and $A_{2 A}$ receptor dual antagonist, 5-[5-amino-3-(4-fluorophenyl) pyrazin-2-yl]-1-isopropylpyridine-2(1H)-one (ASP5854), in models of Parkinson's disease and cognition. J Pharmacol Exp Ther 2007; 323: 708-19.

48. Queiroz G, Talaia C, Goncalves J. Adenosine $A_{2 A}$ receptormediated facilitation of noradrenaline release involves protein kinase $\mathrm{C}$ activation and attenuation of presynaptic inhibitory receptor-mediated effects in the rat vas deferens. J Neurochem 2003; 85: 740-8. 